\title{
PREVALÊNCIA DO TABAGISMO ENTRE ACADÊMICOS DE MEDICINA DE UMA UNIVERSIDADE DO INTERIOR DO ESTADO DE SÃO PAULO
}

Jessyka Bertoldo Lopes, Tatiane Bordignon Uliana, Heloisa Stang Huning, Lilian Francisco Arantes de Souza, Emanuele Moraes Mello

Universidade do Oeste Paulista - UNOESTE, Faculdade de Medicina, Presidente Prudente, SP. E-mail: jessykabertoldo@hotmail.com

\section{RESUMO}

O objetivo deste estudo foi verificar a prevalência de fumantes entre acadêmicos do curso de medicina. Dentre 455 alunos que responderam ao questionário, a prevalência de tabagistas foi de $8,1 \%$, sendo a média das idades de 24,9 anos e predomínio masculino $(64,8 \%, p=0,0004)$. Os principais motivos do início tabágico foram: amigos (45,9\%), ansiedade $(32,4 \%)$ e estresse (18,9\%). Predominantemente moravam sozinhos $(45,9 \%)$ e conviviam com fumantes $83,7 \% \quad(p<0,0001)$. Os profissionais de saúde eram considerados como "modelos de comportamento" para $70 \%$ dos tabagistas e não tabagistas, entretanto, os não tabagistas julgam com maior importância a realização de campanhas sobre o assunto $(p=0,0183)$. 0 grau leve de dependência caracterizou $89,1 \%$ dos tabagistas, conforme o Teste de Fagerstöm. Apesar de $72,9 \%$ relatarem o desejo de abandonar o tabagismo, somente $48,6 \%$ tentaram e não obtiveram êxito. Como conclusão, foi encontrada prevalência de fumantes relativamente baixa, comparado com estatísticas nacionais.

Palavras-chave: hábito de fumar, transtorno por uso de tabaco, dependência, educação médica, estudantes de medicina.

\section{PREVALENCE OF SMOKING AMONG MEDICAL STUDENTS OF A UNIVERSITY IN THE INTERIOR OF THE STATE OF SÃO PAULO.}

\begin{abstract}
The objective of this study was to verify the prevalence of smokers among medical students. Among the 455 students who answered the questionnaire, the prevalence of smokers was $8.1 \%$, the mean age being 24.9 years and male predominance $(64.8 \%, \mathrm{p}=0.0004)$. The main reasons for smoking initiation were: friends $(45.9 \%)$, anxiety $(32.4 \%)$ and stress (18.9\%). Predominantly they lived alone $(45.9 \%)$ and keep social relationwith smokers $83.7 \%(\mathrm{p}<0.0001)$. Health professionals were considered as "behaviour models" for $70 \%$ of smokers and non-smokers, however, non-smokers consider it more important to conduct campaigns on the subject $(p=0.0183)$. The mild degree of dependence characterized $89.1 \%$ of smokers, according to the Fagerstöm Test. Although $72.9 \%$ reported their desire to quit smoking, only $48.6 \%$ tried, and unsuccessfully. As a conclusion, smoking prevalence was relatively low compared to national statistics. Keywords: smoking habit, tobacco use disorder, dependency, medical education, medical students.
\end{abstract}

\section{INTRODUÇÃO}

No passado o tabagismo era visto como um estilo de vida e, por cerca de três séculos, os próprios médicos foram seus maiores divulgadores, por considerarem-no uma droga terapêutica ${ }^{1}$. Atualmente é a principal causa de enfermidades evitáveis ${ }^{2}$, representando um problema de saúde pública, além de estar sendo considerada uma doença pediátrica, pois a idade média da iniciação é 15 anos $^{3}$.

Adicionalmente, o levantamento nacional sobre o uso de álcool, tabaco e outras drogas entre universitários das 27 capitais brasileiras determina que a média do início do hábito é de 16 anos, e que a faixa etária mais prevalente é acima de 35 anos $^{4}$. Dados do Instituto Nacional do Câncer indicam que, atualmente, $16 \%$ da 
população adulta brasileira é fumante e que os homens apresentam uma prevalência maior que as mulheres ${ }^{3}$.

Contudo, os profissionais de saúde, tem papel significante no combate à essa epidemia. Isso se confirma por meio de diversos estudos clínicos disponíveis na literatura, onde demonstram que os pacientes seguidores dos conselhos de seu médico para cessação do tabaco, têm de duas a dez vezes mais chances de conseguirem parar de fumar ${ }^{5}$. Ademais, tem se verificado uma negligência ao aconselhamento sobre a necessidade de cessação dessa prática, além de terem deixado de proporcionar assistência aos dependentes. Também tem sido demonstrado que mais de $70 \%$ dos fumantes que desejam interromper o tabagismo gostariam de receber apoio médico para esse propósito ${ }^{6}$.

Diversos estudos têm demostrado aumento na incidência e prevalência do tabagismo no decorrer da faculdade, sendo que dados nacionais indicam uma variação de $10 \%$ a $20 \%^{2,5,7-11}$. Contrariando a tendência mundial, alguns trabalhos apontam redução do número de estudantes de medicina fumantes como nos estudos transversais realizados pela Universidade Federal de Pelotas/RS em que houve uma queda progressiva da prevalência de fumantes encontrada em 1986 (21,6\%), quando comparada com a de $2002(10,1 \%)$, no entanto essas reduções vêm sendo menos visíveis ${ }^{8}$.

O tabagismo representa grande impacto para a morbidade e mortalidade populacional, e no relevante papel dos profissionais da área de saúde, incluindo o médico, não só no aconselhamento insistente, como também na vivência para a cessação do hábito de fumar dos seus pacientes. Logo, este estudo teve como objetivo identificar a prevalência de tabagismo entre os acadêmicos do curso de medicina e correlacionar o hábito de fumar com variáveis epidemiológicas.

\section{MÉTODOS}

Trata-se de um estudo transversal realizado com a participação de 455 alunos devidamente matriculados do primeiro ao último ano do curso de Medicina. Os alunos foram convidados a responder a um questionário autoaplicável, individual e sigiloso, desenvolvido pelos autores deste trabalho. Além disso, foram instruídos a permanecerem nas salas de aula durante o preenchimento a fim de esclarecer eventuais dúvidas e como controle de qualidade do estudo. $O$ estudo foi avaliado e aprovado pelo Comitê de Ética e Pesquisa da UNOESTE (CAAE $\left.n^{\circ} 20868113.6 .0000 .5515\right)$.

No questionário constaram questões que eram direcionadas a todos os estudantes e algumas que se aplicavam apenas aos que se declaravam fumantes. Para todos foram avaliadas variáveis sócio-demográficas (idade, sexo, ano de curso e com quem o estudante reside), exposição ao fumo passivo (presença de fumante na família e convivência com fumante), opinião social quanto os profissionais de saúde, além da importância da realização de campanhas e palestras sobre o assunto.

Para os estudantes que não fumavam, foi perguntado se alguma vez já havia experimentado. Para os tabagistas foram avaliadas questões como: idade de início, motivo que o levou a iniciar, se já experimentou outro tipo de droga, presença de tosse ou chiado no peito, desejo e tentativa de parar, reprova em alguma matéria da faculdade e se já utilizou espaços físicos deste local para o tabagismo. Para estes, também foi aplicado o Teste de Fagerstöm ${ }^{12}$, também autoaplicável, que avalia o grau de dependência em leve, moderado e grave a partir da somatória dos escores.

Os resultados obtidos foram inseridos no programa Microsoft Office Excel $2010^{\circledR}$ para aplicação de estatística descritiva e apresentação. Posteriormente, para avaliar a relação entre o tabagismo e outras variáveis, foram aplicados o teste Qui-quadrado e o teste G. As análises estatísticas foram realizadas no programa Bioestat $5.3^{13}$ e foi considerado nível de significância de $p<0,05$.

\section{RESULTADOS}

$\mathrm{Na}$ Tabela 1 é possível observar diferentes dados quantitativos (frequência absoluta e relativa), investigados nos acadêmicos do curso de medicina. 
Tabela 1. Frequência absoluta ( $\mathrm{n}$ ) e relativa (\%) das variáveis abordadas aos acadêmicos do curso de medicina.

\begin{tabular}{|c|c|c|}
\hline Variável & $\mathbf{n}$ & $\%$ \\
\hline \multicolumn{3}{|l|}{ Sexo } \\
\hline Feminino & 282 & 61,98 \\
\hline Masculino & 173 & 38,02 \\
\hline \multicolumn{3}{|l|}{ Idade (anos) } \\
\hline$<20$ & 50 & 10,99 \\
\hline 20 a 23 & 213 & 46,81 \\
\hline$>23$ & 192 & 42,20 \\
\hline \multicolumn{3}{|l|}{ Reside com } \\
\hline Familiares & 226 & 49,67 \\
\hline Amigos & 79 & 17,36 \\
\hline Sozinho & 150 & 32,97 \\
\hline \multicolumn{3}{|l|}{ Declara-se fumante } \\
\hline Sim & 37 & 8,13 \\
\hline Não & 418 & 91,87 \\
\hline \multicolumn{3}{|c|}{$\begin{array}{l}\text { Não fumante que já fumou ou experimentou } \\
\text { fumar }\end{array}$} \\
\hline Sim & 192 & 45,93 \\
\hline Não & 226 & 54,06 \\
\hline \multicolumn{3}{|c|}{ Tabagismo na família } \\
\hline Sim & 204 & 44,84 \\
\hline Não & 251 & 55,16 \\
\hline \multicolumn{3}{|c|}{ Pai ou mãe tabagistas } \\
\hline Sim & 73 & 16,04 \\
\hline Não & 382 & 83,96 \\
\hline \multicolumn{3}{|c|}{ Convivência diária com fumantes } \\
\hline Sim & 152 & 33,51 \\
\hline Não & 303 & 66,59 \\
\hline \multicolumn{3}{|c|}{$\begin{array}{l}\text { Consideram profissionais da saúde como } \\
\text { "modelo de comportamento" }\end{array}$} \\
\hline Sim & 327 & 71,86 \\
\hline Não & 128 & 21,13 \\
\hline \multicolumn{3}{|c|}{ Importância de campanhas anti-tabagismo } \\
\hline Sem importância & 9 & 1,98 \\
\hline Pouca importância & 27 & 5,93 \\
\hline Importante & 114 & 25,05 \\
\hline Muito importante & 110 & 24,18 \\
\hline Essencial & 195 & 42,86 \\
\hline
\end{tabular}

Os dados quantitativos obtidos apenas dos alunos que se declararam tabagistas (frequência absoluta e relativa), no curso de medicina encontram-se na Tabela 2. É possível observar que dentre os fumantes, $35,1 \%$ eram do sexo feminino e $64,8 \%$ do sexo masculino, sendo obtida diferença estatisticamente significativa $(p=0,0004)$ entre o hábito de fumar e o sexo, mostrando a influência dessas variáveis. A idade média entre os fumantes foi de 24,9 anos, com limites entre 18 e 38 anos, sendo que a que mais predominou foi 23 anos $(13,5 \%)$.

Em relação ao motivo para iniciar o hábito de fumar, $45,9 \%$ dos fumantes declararam que os amigos foram a principal influência, em seguida, sintomas como ansiedade $(32,4 \%)$ e estresse $(18,9 \%)$ foram os principais fatores declarados. Ademais, $78,3 \%$ dos fumantes 
afirmaram usar ou já ter experimentado outro tipo de substância psicoativa, sendo $56,7 \%$ bebida alcoólica, $29,7 \%$ maconha e $13,5 \%$ outras.

Para os sintomas respiratórios, somente 24,3\% declaram apresentar com frequência tosse ou chiado no peito. Por outro lado, $78,3 \%$ declaram já ter reprovado em alguma matéria durante a faculdade de medicina e $51,3 \%$ declaram terem usado espaços físicos da universidade para fumar.

Entre os estudantes tabagistas entrevistados nesse estudo, $72,9 \%$ relataram ter o desejo de abandonar o tabagismo, sendo que
$48,6 \%$ desses referiram já ter passado por diversas tentativas de cessação do tabagismo, sem êxito.

Foi encontrado que a maioria dos tabagistas entrevistados não são considerados fortemente dependentes da nicotina $(89,1 \%)$, sendo que apenas $10,8 \%$ apresentaram ser dependentes de maneira moderada a grave. Quando se analisou a prevalência de tabagismo por ano de graduação, observou-se maior prevalência no segundo e no quarto ano da amostra, ambos com $21,6 \%$ de tabagistas cada.

Tabela 2. Frequência absoluta (n) e relativa (\%) das variáveis abordadas aos acadêmicos tabagistas do curso de medicina.

\begin{tabular}{|c|c|c|}
\hline Variável & $n$ & $\%$ \\
\hline \multicolumn{3}{|l|}{ Sexo } \\
\hline Feminino & 13 & 35,13 \\
\hline Masculino & 24 & 64,86 \\
\hline \multicolumn{3}{|c|}{ Idade atual (anos) } \\
\hline$<20$ & 2 & 5,41 \\
\hline 20 a 23 & 16 & 43,24 \\
\hline$>23$ & 19 & 51,39 \\
\hline \multicolumn{3}{|c|}{ Idade que começou a fumar (anos) } \\
\hline$<20$ & 29 & 78,38 \\
\hline 20 a 23 & 6 & 16,22 \\
\hline$>23$ & 2 & 5,41 \\
\hline \multicolumn{3}{|c|}{ Tempo de tabagismo (anos) } \\
\hline$<3$ & 7 & 18,92 \\
\hline 3 a 10 & 21 & 56,76 \\
\hline$>10$ & 9 & 24,32 \\
\hline \multicolumn{3}{|c|}{ Grau de dependência } \\
\hline Leve & 33 & 89,19 \\
\hline Moderado & 3 & 8,11 \\
\hline Grave & 1 & 2,70 \\
\hline
\end{tabular}

Quando questionados sobre presença de tabagismo na família, a maioria dos fumantes declarou possuir familiares fumantes $(56,7 \%)$ sendo que $33,3 \%$ destes declaram ser tal familiar pai e/ou mãe. De maneira semelhante, não há influência dessas variáveis com hábito de fumar. Além disso, $83,7 \%$ dos fumantes declararam conviver diariamente com indivíduos fumantes. Entre a convivência e o hábito de fumar foi obtida influência estatisticamente significativa $(p<0,0001)$, demonstrando que indivíduos tabagistas convivem mais com tabagistas, quando comparados com aqueles que não apresentam esse hábito.
A Tabela 3 exibe que a maioria dos estudantes tabagistas $(72,9 \%)$ consideram os profissionais de saúde como "modelos de comportamento" para seus pacientes e o público em geral. De forma concordante, os indivíduos não tabagistas também os consideram, não sendo encontrada diferença significativa $(p=0,8761)$ entre essa variável e o hábito de fumar.

Adicionalmente, $83,7 \%$ dos fumantes declararam importante, muito importante ou essencial a realização de campanhas e palestras sobre o assunto na faculdade para o estímulo à cessação do hábito de fumar. No entanto, houve correlação significativa $(p=0,0183)$ entre 
importância de campanhas anti-tabagismo e o hábito de fumar, sendo que os não tabagistas consideram de uma maneira mais importante a sua realização, quando comparados aos tabagistas. Desses, $16,2 \%$ dos tabagistas e $5 \%$ dos não tabagistas consideram com pouca importância e $44 \%$ dos não tabagistas e $29,7 \%$ dos tabagistas consideram essencial.

Tabela 3. Frequência percentual (\%) das variáveis abordadas aos acadêmicos tabagistas e não tabagistas do curso de medicina de uma universidade no interior paulista.

\begin{tabular}{|c|c|c|c|}
\hline Variável & Tabagistas & $\begin{array}{c}\text { Não } \\
\text { tabagistas }\end{array}$ & p valor \\
\hline \multicolumn{4}{|l|}{ Sexo } \\
\hline Feminino & 35,14 & 64,35 & \multirow{2}{*}{$0,0004^{*}$} \\
\hline Masculino & 64,86 & 35,65 & \\
\hline \multicolumn{4}{|l|}{ Idade (anos) } \\
\hline$<20$ & 5,41 & 11,48 & \multirow{3}{*}{$0,3247^{* *}$} \\
\hline 20 a 23 & 43,24 & 47,13 & \\
\hline$>23$ & 51,35 & 41,39 & \\
\hline \multicolumn{4}{|l|}{ Ano da faculdade } \\
\hline 1 & 13,51 & 16,99 & \multirow{6}{*}{$0,7441^{* *}$} \\
\hline 2 & 21,62 & 16,03 & \\
\hline 3 & 18,92 & 16,51 & \\
\hline 4 & 21,62 & 16,27 & \\
\hline 5 & 13,51 & 16,99 & \\
\hline 6 & 10,81 & 17,22 & \\
\hline \multicolumn{4}{|c|}{ Tabagismo na família } \\
\hline Sim & 56,76 & 43,78 & \multirow{2}{*}{$0,1282 *$} \\
\hline Não & 43,24 & 56,22 & \\
\hline \multicolumn{4}{|c|}{ Tabagismo sendo pai e/ou mãe } \\
\hline Sim & 33,33 & 21,66 & \multirow[b]{2}{*}{$0,1880^{*}$} \\
\hline Não & 66,67 & 78,33 & \\
\hline \multicolumn{4}{|c|}{ Convivência com outros tabagistas } \\
\hline Sim & 83,78 & 28,95 & \multirow{2}{*}{$<0,0001^{*}$} \\
\hline Não & 16,22 & 71,05 & \\
\hline \multicolumn{4}{|l|}{ Reside com } \\
\hline Familiares & 35,14 & 50,96 & \multirow{3}{*}{$0,1475^{*}$} \\
\hline Amigos & 18,92 & 17,22 & \\
\hline Sozinho & 45,95 & 31,82 & \\
\hline \multicolumn{4}{|c|}{ Consideram profissionais da saúde como "modelo de comportamento" } \\
\hline Sim & 72,97 & 71,77 & \multirow{2}{*}{$0,8761^{*}$} \\
\hline Não & 27,03 & 28,23 & \\
\hline \multicolumn{4}{|c|}{ Importância de campanhas anti-tabagismo } \\
\hline Sem importância & 0,00 & 2,15 & \multirow{5}{*}{$0,0183 * *$} \\
\hline Pouca importância & 16,22 & 5,02 & \\
\hline Importante & 37,84 & 23,92 & \\
\hline Muito importante & 16,22 & 24,88 & \\
\hline Essencial & 29,73 & 44,02 & \\
\hline
\end{tabular}

*teste qui-quadrado; ${ }^{* *}$ teste $\mathrm{G}$

\section{DISCUSSÃO}

A prevalência de tabagismo encontrada entre os estudantes de medicina da universidade estudada, no interior paulista, foi de $8,1 \%$ sendo, Colloq Vitae 2018 mai-ago; 10(2): 54-58. DOI: 10.5747/cv.2018.v10.n2.v232 ISSN 1984-6436/@ 2018 - Publicado pela Universidade do Oeste Paulista. Artigo Open Access sob uma licença CC BY-NC-ND (http://creativecommons.org/licenses/by-nc-nd/4.0/). portanto, menor que a população geral brasileira $(17,5 \%)$, conforme estudo domiciliar realizado em todas capitais brasileiras ${ }^{14}$, e menor que a população brasileira universitária de todas as 
áreas que faz uso de tabaco $(21,6 \%)$, conforme levantamento realizado em universidades brasileiras ${ }^{4}$.

$\mathrm{Na}$ literatura, estudos recentes com acadêmicos de medicina de outras universidades brasileiras apontam uma prevalência de 9,4\% a $20,2 \%$ de estudantes tabagistas ${ }^{2,5,7-11}$. Ademais, há uma tendência temporal de redução significativa dessa prevalência, como determina estudos transversais realizados pela Universidade Federal de Pelotas/RS em que houve uma queda progressiva da prevalência de fumantes encontrada em $1986(21,6 \%)$, quando comparada com a de $2002(10,1 \%)^{8}$.

Nesse estudo foi encontrada maior prevalência significativa de tabagistas do sexo masculino $(64,8 \%)$, apesar de a amostra ter maior participação feminina (61,9\%). Esses dados corroboram a maioria dos estudos encontrados na literatura ${ }^{4,5,7,8,10,15,16}$, exceto os resultados obtidos nos trabalhos de Magliari et al. ${ }^{2}$ e de Zettler et al. ${ }^{9}$, com respectivos $60,5 \%$ e $55,3 \%$ de prevalência de mulheres tabagistas. No entanto, além da participação superior de sexo feminino nestes estudos, segundo Silva Junior et al. ${ }^{7}$, estudos recentes mostram que enquanto 0 número de mulheres fumantes aumenta consideravelmente, há um decréscimo do número de homens fumantes.

Adicionalmente, foram observados que $45,9 \%$ dos não fumantes já fumou ou experimentou fumar, sendo esse um número alto e preocupante devido à possiblidade viciosa do tabaco e suas complicações mesmo na primeira tragada. Um estudo na Universidade da Beira Interior em Portugal ${ }^{16}$ mostrou índice maior que o estudo presente ao encontrar que $62,2 \%$ dos alunos entrevistados não fumantes já experimentaram fumar.

Outra condição alarmante refere-se ao início precoce do tabagismo, sendo que neste estudo $78,3 \%$ dos fumantes iniciaram o hábito de fumar antes dos 20 anos, o que corrobora com dados brasileiros onde a idade média da iniciação varia de 15 a 16 anos $^{3,4}$. Além disso, a faixa etária mais prevalente dos fumantes no momento deste estudo foi dos 20 aos 23 anos $(43,2 \%)$ com idade média de 24,9 anos, um pouco acima das idades médias dos fumantes nos estudos presentes na literatura, os quais variam de 21,6 a 22,6 $\operatorname{anos}^{2,5,7,8,16}$.

Os autores observaram que $56,7 \%$ dos estudantes tabagistas têm duração de hábito fumar de 3 a 10 anos, dados equiparáveis aos encontrados por Magliari et al. ${ }^{2}$, que encontraram uma duração de 4 a 10 anos em $54 \%$ dos estudantes fumantes. Adicionalmente, nesse estudo foi observado que o número de tabagistas é discretamente maior no segundo e no quarto ano de curso (21,6\% cada e sem significância estatística). Menezes et al. ${ }^{8}$ demonstraram uma tendência linear direta entre tabagismo e ano cursado mesmo ajustando os dados para a idade. Embora as razões para o aumento da prevalência conforme $o$ ano cursado não sejam claras, Menezes et al. $^{8}$ sugere que a possiblidade desse resultado seja conhecimento ineficaz repassado aos alunos e modelos de comportamento e de convívio inadequados.

A influência social para iniciar o hábito de fumar também foi analisada, revelando que neste estudo $37,8 \%$ dos fumantes relataram serem os amigos o maior motivo para começar a fumar. No estudo de Marin et al. ${ }^{5}$ os amigos foram responsáveis por 46,3\%, também sendo considerado como principal motivo de iniciar o hábito de fumar, seguido por integração social $(19,4 \%)$ e por influência familiar $(14,9 \%)$. No presente estudo, a integração social representou $2,7 \%$ e a influência familiar não foi relatada pelos estudantes. No entanto, $56,7 \%$ dos fumantes relatam possuir fumantes na família sendo que $33,3 \%$ desses relataram tal familiar ser pai e/ou mãe. Nos estudos de Silva Junior et al. ${ }^{7}$ e de Menezes et al. ${ }^{8}$, o tabagismo entre pais é de $29 \%$ e $22,2 \%$ respectivamente. Contudo, o tabagismo na família e dos pais não apresentou significância estatística nesse estudo, assim como o estudo de Silva Junior et al. ${ }^{7}$ em que a correlação tetracórica entre tabagismo dos pais e dos alunos foi qualitativamente fraca e negativa.

Sintomas como ansiedade (27\%), estresse $(16,2 \%)$ e depressão $(2,7 \%)$ foram relatados no atual estudo como motivos para iniciar o tabagismo. Ademais, $45,9 \%$ dos tabagistas relataram morar sozinhos. No estudo de Zettler et al. ${ }^{9} 46,4 \%$ dos tabagistas relataram ter sintomas de ansiedade e depressão e $50 \%$ dos tabagistas morar sozinhos, o que poderia levantar a hipótese de a presença de sintomas como ansiedade, estresse e depressão aliada a solidão atuar como potencial fator de risco.

Contudo, analisando o convívio diário dos tabagistas, pode-se observar significância estatística entre esses e os não tabagistas, onde $83,7 \%$ dos tabagistas relataram conviver com 
outros fumantes, revelando um alto índice de correlação de convívio com hábito fumar dos alunos de medicina tabagistas. Nos estudos de Marin et al. ${ }^{5}$ e Carvalho $^{16}$ esse convívio dos tabagistas com outros tabagistas é de $32,5 \%$ e $32,8 \%$ respectivamente.

A relação entre tabagismo e uso de outras substâncias psicoativas também foi analisada, sendo que neste estudo $56,7 \%$ dos tabagistas já fizeram ou fazem uso frequente de álcool, $29,7 \%$ de maconha e $13,5 \%$ de outras substâncias psicoativas. No estudo de Buzatto e Soler $^{11}, 57,7 \%$ dos acadêmicos de medicina da Faculdade de Medicina de São José do Rio Preto referiram ingerir álcool frequentemente, sendo que no estudo de Petroianu et al. ${ }^{10}$ o consumo de álcool, tabaco, maconha e alucinógenos é de $85,2 \%, 16,3 \%, 16,5 \%$ e $6,9 \%$ respectivamente, entre os acadêmicos de medicina da Universidade Federal de Minas Gerais. No levantamento entre universitários de todas as áreas de estudo realizado pelo Ministério da Saúde ${ }^{4}$, as drogas relatadas com maior frequência foram: álcool $(86,2 \%)$, tabaco $(46,7 \%)$ e maconha (26,1\%). No estudo de Menezes et al. ${ }^{8} 6,3 \%$ dos acadêmicos de medicina da Universidade Federal de Pelotas utilizou maconha alguma vez, sendo que os mesmos autores realizaram estudo semelhante 8 anos antes e esse índice foi de $4,2 \%$. Esses dados comprovam que o consumo de álcool, tabaco e entorpecentes podem estar correlacionados, sendo um problema de saúde pública o que estimula vários estudos e iniciativas a respeito.

Observou-se neste estudo relação positiva entre o mau desempenho escolar, mensurado através da reprova em pelo menos uma disciplina da faculdade, e o tabagismo, sendo que $78,3 \%$ dos fumantes relataram tal episódio. Zettler et al. ${ }^{9}$ obtiveram dados semelhantes, mas em um número menos expressivo $(44,6 \%)$, podendo, o sucesso escolar, representar um fator de proteção para a aquisição do hábito tabágico entre os estudantes.

Em relação aos sintomas respiratórios (tosse ou chiado no peito), os mesmos estão presentes em $24,3 \%$ dos fumantes desse estudo. Apesar dessa informação ser escassa na literatura, Menezes et al. ${ }^{8}$ abordaram esse tópico em seu trabalho, no entanto, o autor reconhece que a causalidade reversa pode afetar a associação tabagismo com sintomas respiratórios, pois tanto o tabagismo pode provocar os sintomas respiratórios como os sintomas desses pode não estar relacionado ou até impedir o tabagismo.

Foi observado nesse estudo que $72,9 \%$ dos fumantes têm desejo de parar de fumar, sendo esse um passo essencial para o sucesso do tratamento e cessação do tabagismo. Ademais, $48,6 \%$ dos fumantes já tentaram parar de fumar sem sucesso. $\mathrm{Na}$ literatura esses índices são semelhantes, sendo que nos estudos de Marin et al. ${ }^{5}$, Magliari et al. ${ }^{2}$, Carvalho ${ }^{16}$ e Silva Junior et al. ${ }^{7}$ os estudantes tabagistas que desejam parar representam 58,2\%, 58,1\%, 92,5\% e $77 \%$ respectivamente; e os que já tentaram sem sucesso têm índices de 64,2\%, 46,5\%, 47,2\% respectivamente, sendo que Silva Junior et al. ${ }^{7}$ não abordou essa última variável. Magliari et al. ${ }^{2}$ relata ser comum que haja diversas tentativas antes do indivíduo conseguir interromper o hábito com sucesso. Concordando com essa dificuldade, Menezes et al. $^{8}$ e Zettler et al. ${ }^{9}$ mostraram número discreto de ex-tabagistas, respectivamente de $9,8 \%$ e $7,3 \%$. Pôde-se observar ainda que a maioria dos acadêmicos de medicina tabagistas deste estudo apresentam dependência à nicotina de grau leve $(89,1 \%)$, corroborando com os estudos de Marin et al. ${ }^{5} \mathrm{e}$ Silva Junior et al. ${ }^{7}$, em que $65 \%$ e $73,1 \%$ respectivamente dos alunos apresentaram grau leve de dependência à nicotina, utilizando também o Teste de Fagerström. Haggsträm et al. ${ }^{17}$ mostraram que o alto grau de dependência à nicotina está associada ao maior fracasso no tratamento de cessação do tabagismo. Assim, pode-se considerar que com a adoção de medidas de cessação do hábito de fumar entre a população de grau leve de dependência a nicotina, como se apresentam os alunos tabagistas do interior paulista, poderiam ser obtidos resultados satisfatórios. Em desacordo com esses dados, o Levantamento nacional encontrou maior prevalência de dependência de nicotina em universitários de instituições privadas, para os cursos de Ciências Biológicas, de período integral ${ }^{4}$. Contudo, poderia ser questionado o grau de confiabilidade em responder o questionário, por parte dos acadêmicos, tendo em vista que quase $50 \%$ desses já tentaram abandonar o tabagismo, sem êxito.

Apesar da legislação federal que proíbe fumar em ambientes coletivos fechados ter representado um grande avanço no controle do 
tabaco, ainda se permite fumar em áreas reservadas nos recintos coletivos. Sendo assim, $51,3 \%$ dos acadêmicos de medicina relataram usar os espaços físicos da faculdade para praticar o fumo. Em estudo realizado por Szklo et al. ${ }^{15}$ com universitários de odontologia, medicina, enfermagem e farmácia da cidade do Rio de Janeiro demonstrou que esse índice é de $80 \%$. Szklo et al. ${ }^{15}$ ainda propõe ser possível que os universitários estejam fumando em áreas reservadas para fumantes ou não estejam cumprindo a lei. De qualquer forma, pessoas não fumantes poderiam estar sendo expostas, inclusive os pacientes dos hospitais universitários, devendo-se reforçar a necessidade de fiscalização. Carvalho ${ }^{16}$ ressalta que a população não fumante que permanece exposta à fumaça do tabaco ambiental têm maior probabilidade de desenvolver câncer de pulmão, doenças cardiovasculares, doenças respiratórias e infecções, quando comparadas à população não exposta.

Quando questionados sobre a importância da realização de campanhas e palestras sobre o estímulo à cessação do tabagismo na faculdade, ambos acadêmicos, tabagistas e não tabagistas concordaram com a sua realização. Entretanto, encontrou-se significância $(p=0,0183)$ quanto ao grau de importância dessa realização, sendo que aqueles não tabagistas consideram de uma maneira mais importante a sua realização, quando comparados aos tabagistas. Os resultados obtidos nesse trabalho estão em desacordo com os obtidos nos estudos de Carvalho ${ }^{16}$ e de Szklo et al. $^{15}$, que declararam uma importância de $99,4 \%$ e mais de 90\% respectivamente, para os acadêmicos tabagistas. Dessa forma, os acadêmicos fumantes estudados estão mais desacreditados quanto a importância de informações antitabagicas do profissional de saúde em relação aos demais acadêmicos previamente estudados, necessitando assim salientar maiores esforços instrucionais de campanhas antitabagismo nesse grupo de acadêmicos.

Apesar disso, mais de $70 \%$ dos acadêmicos de medicina desse estudo, tabagistas e não tabagistas, consideram os profissionais de saúde como "modelos de comportamento" para os pacientes e o público em geral. Na literatura, $80 \%$ dos acadêmicos do estudo de Carvalho ${ }^{16}$ e $77 \%$ dos acadêmicos do estudo de Szklo et al. ${ }^{15}$ também consideram os profissionais de saúde como "modelo de comportamento". Assim sendo, é possível que a credibilidade do profissional de saúde, especialmente o médico, junto ao paciente fumante, contribua para a eficácia do tratamento ou da mensagem que está sendo transmitida.

A prevalência de tabagismo entre os acadêmicos de medicina da universidade estudada é de $8,1 \%$, menor quando comparada com outras faculdades de medicina e com estatísticas nacionais. As variáveis com influência estatisticamente significativa sobre o hábito de fumar foram sexo, convivência com outros tabagistas e a opinião acerca de campanhas de estímulo a cessação da prática, sendo que os não tabagistas consideram de uma maneira mais importante a sua realização, quando comparados aos tabagistas. Os autores reforçam que o conhecimento dos dados de tabagismo entre os estudantes de medicina representa um aliado essencial aos esforços, tanto por parte do núcleo docente acadêmico em relação às campanhas de cessação do tabagismo em toda a comunidade, quanto aos futuros profissionais médicos, que serão os principais agentes na promoção de saúde e diminuição das taxas de tabagismo na população em geral. Para isso, os autores sugerem que as universidades incorporem políticas antitabaco aos currículos dos cursos universitários, assim como estimulem a realização de pesquisas envolvendo os estudantes, a fim de avaliar a prevalência do tabagismo, sobretudo, na faculdade de medicina.

\section{CONFLITO DE INTERESSE}

Os autores declaram não haver qualquer potencial de conflito de interesse que possa interferir na imparcialidade deste trabalho científico.

\section{REFERÊNCIAS}

1. Rosemberg J. Pandemia do tabagismo: enfoques históricos e atuais. São Paulo: Secretaria da Saúde, Centro de Vigilância Epidemiológica; 2002.

\section{Magliari RT, Pagliusi AL, Previero BM, Menezes} FR, Feldman A, Novo, NF. Prevalência de tabagismo em estudantes de faculdade de medicina. Rev Med (São Paulo). 2008;87(4):26471. DOI: https://doi.org/10.11606/issn.16799836.v87i4p264-271 
3. Brasil. Ministério da Saúde. Secretaria de Atenção à Saúde, Instituto Nacional do Câncer. Coordenação de Prevenção e Vigilância. A situação do câncer no Brasil. Rio de janeiro: INCA, 2006.

4. Brasil. Presidência da República. Secretaria Nacional de Políticas sobre Drogas. I Levantamento Nacional sobre o Uso de Álcool, Tabaco e Outras Drogas entre Universitários das 27 Capitais Brasileiras / Secretaria Nacional de Políticas sobre Drogas; GREA/IPQ-HC/FMUSP; organizadores Arthur Guerra de Andrade, Paulina do Carmo Arruda Vieira Duarte, Lúcio Garcia de Oliveira. - Brasília: SENAD, 2010.

5. Marin NS, Rodrigues ART, Kinoshita NGC, Nakamura AS, Bueno PCS, Kinoshita SK. Tabagismo: caracterização do grau de dependência entre estudantes de medicina. RBSP. 2012;36(2):408-17.

6. Viegas CAA, Andrade AA, Silvestre RS. Characteristics of smoking among physicians in the Federal District of Brazil. J Bras Pneumol. 2007;33(1):76-80.

DOI:

http://dx.doi.org/10.1590/S1806-

$\underline{37132007000100015 .}$.

7. Silva Junior CT, Braga UM, Vieira HV. Prevalência de tabagismo entre estudantes de graduação em medicina da Universidade Federal Fluminense. Pulmão RJ. 2006;15(1):11-15.

8. Menezes $A M B$, Hallal PC, Silva $F$, Souza $M$, Paiva $L, D$ ávila $A$, et al. Smoking among medical students: temporal trends and related variables. J Bras Pneumol. 2004;30(3):223-8. DOI: http://dx.doi.org/10.1590/S180637132004000300007.

9. Zettler EW, Nudelmann LM, Cunha DP, Hilgert $C$, Mattos $M D$, Scholl $M$, et al. Prevalência do tabagismo entre estudantes de medicina $\mathrm{e}$ fatores de risco associados. Revista AMRIGS. 2005;49(1):16-9.

10. Petroianu A, Reis DCF, Cunha BDS, Souza DM. Prevalência do consumo de álcool, tabaco e entorpecentes por estudantes de medicina da Universidade Federal de Minas Gerais. Rev Assoc Med Bras. 2010;56(5):568-71. DOI:
http://dx.doi.org/10.1590/S0104$\underline{42302010000500019}$.

11. Buzatto SV, Soler LASG. Tabagismo e etilismo entre acadêmicos de medicina e enfermagem da Faculdade de Medicina de São José do Rio Preto SP - FAMERP. Arquivo de Ciências da Saúde. 2010. 17(3): 122-127.

12. Heatherton TF, Kozlowski LT, Frecker RC, Fagerströn KO. The Fagerström Test for Nicotine Dependence: a revision of the Fagerström Tolerance Questionnaire. Br J Addict. 1991. 86 (9): 1119-27. DOI: https://doi.org/10.1111/j.13600443.1991.tb01879.x.

13. Ayres $M$, Ayres Junior $M$, Ayres DL, Santos AS. Bioestat 5.0 - Aplicações estatísticas nas áreas das ciências biomédicas. ONG Mamiraua, 2007.: 364.

14. BRASIL. Instituto Nacional de Câncer. Ministério da Saúde. Instituto Brasileiro de Geografia e Estatística. Ministério do Planejamento, Orçamento e Gestão. Pesquisa Nacional por Amostra de Domicílios. -Rio de Janeiro, 2008. 128p.

15. Szklo AS, Sampaio MMA, Martins LFL, Fernandes EM, Almeida LM. O Tabagismo no contexto dos futuros profissionais de saúde do Rio de Janeiro. Rev Bras Cancerol. 2011;57(3):321-7.

16.Carvalho LS. Comportamento tabágico e atitudes de controlo de tabagismo dos estudantes de Medicina da Universidade da Beira Interior. Dissertação (Mestrado em Medicina) - Ciências da Saúde, Universidade de Beira Interior, Covilhã; 2012.

17. Haggsträm FM, Chatkin JM, Cavalet-Blanco D, Rodin V, Fritsher CC. Tratamento do tabagismo com bupropiona e reposição nicotínica. J Penumol. 2001;27(5):255-61. DOI: http://dx.doi.org/10.1590/S0102$\underline{35862001000500005}$.

Recebido para publicação em 30/01/2017

Revisado em 22/03/2018

Aceito em 08/08/2018 